\title{
Downregulation of $\beta 3$ integrin by miR-30a-5p modulates cell adhesion and invasion by interrupting Erk/Ets-1 network in triple-negative breast cancer
}

\author{
WENTONG LI ${ }^{1}$, CHUANLIANG LIU ${ }^{2}$, CHUNLING ZHAO ${ }^{3}$, LIMIN ZHAI ${ }^{1}$ and SHIJUN LV ${ }^{1,2}$ \\ ${ }^{1}$ Department of Pathology, Weifang Medical University, Weifang, Shandong 261053; \\ ${ }^{2}$ The Third Department of Health Care, Weifang People's Hospital, Weifang, Shandong 261053; \\ ${ }^{3}$ Department of Medical Biology, Weifang Medical University, Weifang, Shandong 261053, P.R. China
}

Received November 19, 2015; Accepted December 15, 2015

DOI: $10.3892 /$ ijo.2016.3319

\begin{abstract}
Integrins are adhesion receptors involved in bidirectional signaling and are crucial for various cellular responses during normal homeostasis and pathological conditions, such as cancer progression and metastasis. In the present study, we demonstrated that blockage of $\beta 3$ integrin-mediated cellextracellular matrix interactions restrained triple-negative breast cancer (TNBC) growth, and elevated $\beta 3$ integrin can trigger the rewiring of Erk/Ets-1 signaling pathways, thereby enhancing cell growth and invasion. Ectopic expression of miRNA has been implicated in the deregulation of integrin expression and activity, blocking of cancer tumor development and progression, and acquisition of metastatic phenotype. miR-30a-5p expression has been implicated in the progression of breast cancer. Overexpression of miR-30a-5p suppressed the proliferation, migration and invasion of breast cancer cells. On the contrary, inhibition of miR-30a-5p promoted the proliferation, migration, and invasion of TNBC cells by suppressing the expression of ERK/Ets-1 signal. An inverse correlation was found between the mRNA expressions of miR-30a-5p and $\beta 3$ integrin in TNBC samples. Furthermore, bioinformatics analysis revealed the putative miR-30 binding sites in the 3'-UTR of $\beta 3$ integrin. Results of luciferase assay revealed a strong repression of luciferase activity after transfection with miR-30a-5p and wild-type 3'-UTR of $\beta 3$ integrin. In TNBC cells, miR-30a-5p promoted an epithelial phenotype and suppressed invasion by specifically targeting $\beta 3$ integrin subunit to subsequently interdict the $\beta 3$ integrin/Erk/Ets-1 network.
\end{abstract}

Correspondence to: Dr Wentong Li, Department of Pathology, Weifang Medical University, Weifang, Shandong 261053, P.R. China E-mail: liwentong11@163.com

Key words: miR-30a-5p, $\beta 3$ integrin, triple-negative breast cancer, ERK, invasion

\section{Introduction}

Triple-negative breast cancer (TNBC) is a highly aggressive subcategory of breast cancer that currently lacks well-defined molecular targets for effective targeted therapies. Disease relapse, metastasis and drug resistance render standard chemotherapy ineffective in the treatment of TNBC. The acquisition of metastatic phenotypes by mammary tumors has been linked to the alterations in integrin expression $(1,2)$. High level expressions of many integrins, including $\alpha 5 \beta 1$, $\alpha 6$ and $\alpha v \beta 3$ have been correlated with tumor progression $(3,4)$.

$\beta 3$ integrin is frequently overexpressed in tumor cells, including lung cancer, melanoma, glioblastoma and breast cancer cells $(5,6)$. Previous studies coupled $\beta 3$ integrin to epithelial-mesenchymal transition (EMT) and metastasis; $\beta 3$ integrin inhibition is a therapeutic target to treat TNBC, attenuates TGF- $\beta$-mediated EMT and invasion, and inhibits 3 -dimensional organoid growth (7). $\beta 3$ integrin-mediated adhesion can trigger the activation of numerous signaling intermediates, such as FAK, Src, ILK, PI3K and MAPK $(8,9)$. Evidence that ERK signaling promotes cell proliferation, cell survival and metastasis and that this pathway is aberrantly activated in breast cancer at an overwhelming frequency support current efforts to identify inhibition strategies for this pathway. Thus, finding out whether alterations in $\beta 3$ integrin affect ERK signaling in tumor cells is crucial to improve strategies for treating or preventing metastatic disease.

MiRNAs are powerful regulators of gene expression in cancer cell invasion and metastasis that downregulate gene expression at the post-transcriptional level (10-12). miR-30a has been identified as one of the crucial regulators for development and progression of breast and prostate cancers by directly targeting MTDH and ERG, respectively $(13,14)$. However, the exact function and underlying mechanisms of miR-30a in the progression of breast cancer still warrant further investigation. In the present study, we focused on the functional analysis of miR-30a-5p, a member of the miR-30 family that is reportedly downregulated in cancer cells.

Our data suggested that the activation of signaling cascades downstream from $\beta 3$ integrin involved the ERK/Est-1 pathway. 
Results also showed that miR-30a-5p suppressed the proliferation and invasion of breast cancer cells in vitro by directly targeting the $\beta 3$ integrin and suspended $\beta 3$ integrin-Erk-Ets-1 loop. Thus, a tumor suppressor role of miR-30a-5p in breast cancer was suggested.

\section{Materials and methods}

Patient samples. Breast cancer specimens were obtained from 156 patients at the Weifang Medical University Affiliated Hospital after surgical resection. Twenty para-cancerous tissues were allocated into the negative control group. No patient in the present study received chemotherapy or radiation therapy prior to surgery. This study was approved by the Institutional Review Board of Weifang Medical University Hospital and informed consent was obtained from each patient. All fresh samples were stored at $-80^{\circ} \mathrm{C}$.

Immunohistochemistry, immunofluorescence and cytoskeletal staining. Labeled streptavidin biotin method was used for immunohistochemistry. After deparaffinization and rehydration, primary antibodies were added for overnight storage at $4^{\circ} \mathrm{C}$, and slides were incubated with biotin-labeled secondary antibodies. Finally, the slides were incubated with HRP-streptavidin for $15 \mathrm{~min}$. After DAB staining, the results were graded for intensity $(0,1,2$ and 3 for negative, weak, moderate and strong, respectively). The percentage of positive cells, i.e., 0 and 1 (1-24\%), 2 (25-49\%), $3(50-74 \%)$ and 4 (75-100\%), was determined. Discrepancies were resolved by consensus. The grades were multiplied to determine the scores. Tumor scores were defined by using the following rules: low (score, 0-4) and high (score $\geq 5$ ). For immunofluorescence, cells were grown on coverslips, fixed in $4 \%$ paraformaldehyde, and incubated in a blocking buffer (1\% BSA, $0.25 \%$ Triton X-100 in PBS, pH 7.4). The cells were then probed with primary antibody and fluorescein-conjugated goat anti-mouse IgG (Beyotime Institute of Biotechnology, Haimen, China). The cells were counterstained with DAPI to label the cell nuclei. Cell cytoskeleton was stained with FITC-phalloidin. The cells were seeded into 24-well culture plates, washed with PBS, fixed with $4 \%$ paraformaldehyde and incubated with $0.2 \%$ Triton X-100. After blocking with $1 \%$ bovine serum albumin, cells were incubated with CY3-phalloidin. Images were captured by confocal fluorescent microscopy.

Cell lines and culture conditions. Breast cancer cell lines MCF-7, MDA-MB-231 and MDA-MB-468 were obtained from the American Type Culture Collection (ATCC; Manassas, VA, USA) and were routinely cultured in Dulbecco's modified Eagle's medium (DMEM; Gibco Laboratories, Grand Island, NY, USA) supplemented with $10 \%$ FBS (Tianjin Hao Yang Biological Manufacture Co., Ltd., Tianjin, China). All cells were cultured at $37^{\circ} \mathrm{C}$ and $5 \% \mathrm{CO}_{2}$.

Plasmid construction and transfection/infection. The sequences of miR-30a-5p mimic and mock were synthesized according to the method of Baraniskin et al (15) and were ligated into the restriction sites of pCDH-CMV-MCS-EF1-Puro vectors. Lentiviruses were produced by transfecting human embryonic kidney 293T with a 3-plasmid system according to manual instructions. miR-30a-5p inhibitor was synthesized by Guangzhou RiboBio Co., Ltd. (Guangzhou, China). For $\beta 3$ integrin overexpression, the cDNA of $\beta 3$ integrin was cloned into the pcDNA3.1, as previously described, and transfected into human breast cancer cells using Lipofectimine according to the manufacturer's instructions (16). Total RNA and protein were collected for 2 days post-transfection or viral infection assay.

Quantitative real-time PCR analysis. Total RNA was isolated using TRIzol (Invitrogen, Carlsbad, CA, USA), and complementary DNA was synthesized using reverse transcriptase (Sangon Biotech Co., Ltd., Shanghai, China). Real-time quantitative PCR reactions were performed using SYBR-Green (Takara Bio, Dalian, China). To analyze mature miR-30a-5p, quantitative PCR (RT-qPCR) was performed using the miScript PCR System (Qiagen, Hilden, Germany). The mRNA levels of $\beta 3$ integrin, E-cadherin, vimentin, and Zeb1 were quantified by qRT-PCR using QuantiTect SYBR-Green PCR kit (Vazyme Biotech Co., Ltd., Nanjing, China). Primers used are described in Table I. Changes in expression were calculated using the $\Delta \Delta \mathrm{Ct}$ method. We calculated the median expression value from signal values $\left(\log _{2}\right)$. Patients were labeled based on higher or lower $\beta 3$ integrin expression compared with the median value, as follows: individuals with low $\beta 3$ integrin expression $(<$ median) and those with high $\beta 3$ integrin expression ( $\geq$ median).

Cell proliferation assay and flow cytometric analysis. Cell proliferation was measured via methyl-thiazolyltetrazolium (MTT) assay. Cells were seeded at a density of $5 \times 10^{3} /$ well into 96-well plates and cultured for 24, 48, 72 and $96 \mathrm{~h}$. The cells were then incubated with $20 \mu \mathrm{l}$ MTT $(5 \mathrm{mg} / \mathrm{ml})$ for $4 \mathrm{~h}$ at $37^{\circ} \mathrm{C}$, and $150 \mu l$ dimethyl sulfoxide was added to solubilize the crystals for $10 \mathrm{~min}$ at room temperature (RT). The optical density was measured at $540 \mathrm{~nm}$. For cell cycle analysis, the adhered cells were collected by trypsinization at $48 \mathrm{~h}$ after transfection. The cells were incubated with propidium iodide $(0.05 \mathrm{mg} / \mathrm{ml}$; Sigma) and RNase A ( $0.1 \mathrm{mg} / \mathrm{ml}$; Sigma) for $30 \mathrm{~min}$ at RT in the dark and analyzed by using BD FACSCalibur flow cytometer and CellQuest software.

Adhesion, wound healing and invasion assays. Cells $\left(0.5 \times 10^{6}\right.$ cells/well) were added to each well with $5 \% \mathrm{CO}_{2}$ and incubated for $4 \mathrm{~h}$ at $37^{\circ} \mathrm{C}$. After washing, the attached cells were fixed with $70 \%$ ethanol followed by staining with $0.1 \%$ crystal violet in $20 \%$ ethanol. The stained crystal violet was dissolved in $10 \%$ acetic acid, and the absorbance value was measured at $597 \mathrm{~nm}$. The cell matrix adhesion index was calculated as the OD value (test-negative control)/OD value (positive control-negative control). Each test group was assayed in triplicate and repeated at least thrice. For the scratch wound healing assay, cells were cultured in a serum-free medium for $24 \mathrm{~h}$ and wounded with pipette tips. Wound closing procedure was observed for $48 \mathrm{~h}$ with images taken every $24 \mathrm{~h}$. For the invasion assay, cell invasion through a 3D extracellular matrix (ECM) was assessed using BD Matrigel invasion chambers ((BD Biosciences, Bedford, MA, USA) with $8.0 \mu \mathrm{m}$ filter membranes. After $24 \mathrm{~h}$, cells invading the lower surface of the filters were fixed, stained and counted. Percentage change 
Table I. The primers or oligonucleotides.

\begin{tabular}{|c|c|}
\hline Genes & Primers or oligonucleotides \\
\hline mir-30a-5p mimic & $\begin{array}{l}\text { Sense: 5'-ccggcttccagtcgaggatgtttacactcgagtgtaaacatcctcgactggaagttttg-3' } \\
\text { Antisense: 5'-aattcaaaaacttccagtcgaggatgtttacactcgagtgtaaacatcctcgactggaag-3' }\end{array}$ \\
\hline mir-30a-5p scramble sequence & $\begin{array}{l}\text { Sense: 5'-ctagaggagctccaccgeggtggcatcgatggagctccaccgcggtggcatggtac-3' } \\
\text { Antisense: 5'-catgccaccgcggtggagctccatcgatgccaccgcggtggagctcct-3' }\end{array}$ \\
\hline E-cadherin & $\begin{array}{l}\text { Forward: 5'-accattaacaggaacacagg-3' } \\
\text { Reverse: 5'-cagtcactttcagtgtggtg-3' }\end{array}$ \\
\hline Vimentin & $\begin{array}{l}\text { Forward: 5'-gacctctacgaggaggagat-3' } \\
\text { Reverse: 5'-tccaccaccctgttgctgta-3' }\end{array}$ \\
\hline Zeb1 & $\begin{array}{l}\text { Forward: 5'-agcagtgaaagagaagggaatgc-3' } \\
\text { Reverse: 5'-ggtcctcttcaggtgcctcag-3' }\end{array}$ \\
\hline$\beta 3$ integrin & $\begin{array}{l}\text { Forward: 5'-ctgtatccagccgggctcctatg-3' } \\
\text { Reverse: 5'-gccccggtacgtgatattggtgaa-3' }\end{array}$ \\
\hline $\operatorname{mir}-30 a-5 p$ & $\begin{array}{l}\text { Forward: 5'-gccgctgtaaacatcctacact-3' } \\
\text { Reverse: 5'-gtgcagggtccgaggt-3' }\end{array}$ \\
\hline U6 & $\begin{array}{l}\text { Forward: 5'-ctcgcttcggcagcaca-3' } \\
\text { Reverse: 5'-aacgcttcacgaatttgcgt-3' }\end{array}$ \\
\hline$\beta$-actin & $\begin{array}{l}\text { Forward: 5'-cctgtacgccaacacagtgc-3' } \\
\text { Reverse: 5'-atactcctgcttgctgatcc-3' }\end{array}$ \\
\hline
\end{tabular}

during invasion was determined by counting the number of cells that migrated to the lower surface of the filters. Three separate microscopic fields were counted per membrane.

$\beta 3$ integrin 3'-UTR reporter analysis. The 3'-UTR of $\beta 3$ integrin containing a putative miR-30a-5p binding site was amplified and cloned into pGL3 vector to generate the wild-type construct. An overlap extension PCR assay was used for mutant plasmids, as previously described (17). Cells were cultured in 24-well plates. For the transfection complex, $2 \mu 1$ of $20 \mu \mathrm{M}$ chemically synthesized miR-30a-5p mimic, $150 \mathrm{ng}$ pGL3 reporter plasmid, and $50 \mathrm{ng}$ pRL-TK plasmid were mixed with Lipofectamine 2000. Luciferase activities were measured according to the manufacturer's instructions (DualLuciferase assay system; Promega). Renilla luciferase activity was normalized to corresponding firefly luciferase activity and was plotted as a percentage.

Western blot analysis. Cells were lysed in RIPA buffer. Proteins were separated by using SDS-PAGE and transferred to nitrocellulose. The blots were probed with primary antibodies and incubated with 1:5,000 secondary antibody. Signals were detected with enhanced chemiluminescence. Images were analyzed by the Gel-Pro-Analyzer software. The membranes were stripped and probed with monoclonal antibody for $\beta$-actin as loading control.

Statistical analysis. SPSS version 20.0 software was used for statistical analysis. Student's t-test, Chi-squared test and one-way ANOVA analysis were used to determine significance. $\mathrm{P}<0.05$ was considered to indicate a statistically significant result.

\section{Results}

TNBC expresses low levels of miR-30a-5p and high levels of $\beta 3$ integrin. The expression levels of miR-30a-5p in TNBC breast cancer cell lines (MDA-MB-231 and MDA-MB-486) were much lower than in NTNBC breast cancer cell lines (MCF-7), as shown in Fig. 1A. We compared miR-30a-5p expression levels in para-cancerous tissues of breast cancer and breast cancer patients. Breast cancer tissues had reduced miR-30a-5p transcript levels compared with para-cancerous tissues (Table II for patient characteristics of all donors). Significant differences were observed when comparing TNBC with NTNBC tissues (Fig. 1B and Table II). Further analysis showed that miR-30a-5p expression strongly correlated with histological grade and survival status (Table II).

The $\beta 3$ integrin expression in TNBC patients was compared with that in NTNBC patients. Notably, $\beta 3$ integrin mRNA expression was significantly higher in TNBC patients compared with NTNBC patients and para-cancerous tissues (Fig. 1C) $(\mathrm{P}<0.001)$. Kaplan-Meier analysis was performed by using the log-rank test to calculate the effect of $\beta 3$ integrin mRNA expression on TNBC patient survival. High $\beta 3$ integrin expression was markedly associated with reduced overall survival in TNBC patient subgroups (Fig. 1D). Western blot analyses of protein extracts revealed a significantly higher relative $\beta 3$ integrin expression in TNBC patients (Fig. 1E). Given that miRNAs exploit their inhibitory activity at the posttranscriptional level and the reduced miR-30a-5p expression in the TNBC patients, we subsequently verified the expression of miR-30a-5p and $\beta 3$ integrin in breast tumor tissues. As shown in Fig. 1F, miR-30a-5p expression was lower in TNBC than NTNBC samples. An inverse correlation was found between 

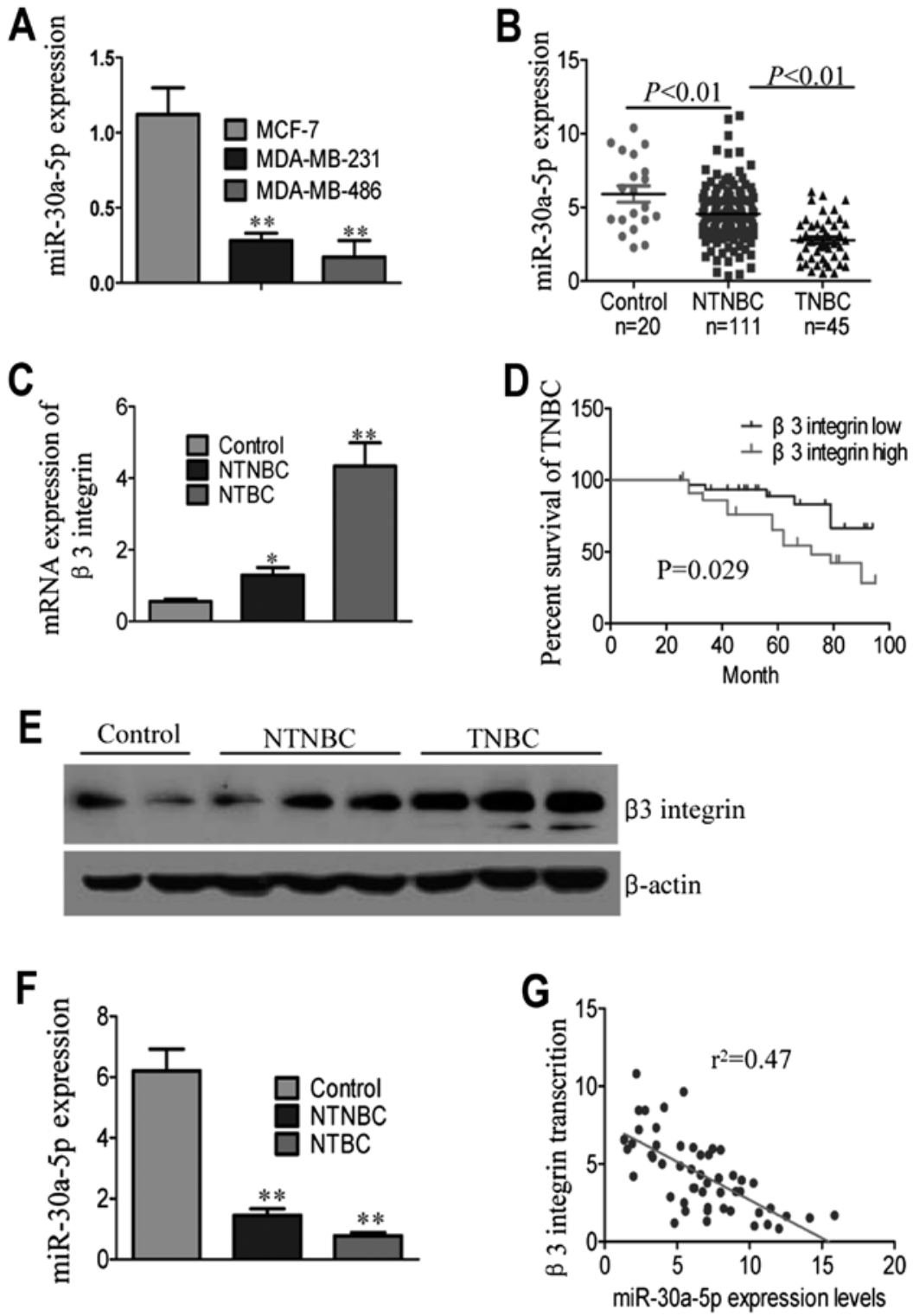

Figure 1. Expression of miR-30a-5p and $\beta 3$ integrin in TNBCs. (A) Expression of miR-30a-5p in breast cancer cell lines was quantitatively analyzed by using qPCR and normalized with U6 snRNA. (B) miR-30a-5p expressions in breast tissue from para-cancerous tissues and breast cancer samples was determined by real-time RT-PCR. (C) $\beta 3$ integrin mRNA expression in breast cancer tissues. (D) Kaplan-Meier curves with univariate analyses (log-rank) for patients with low $\beta 3$ integrin vs. high $\beta 3$ integrin. (E) $\beta 3$ integrin protein expression in para-cancerous tissues and breast cancer samples were determined by western blot analysis. Relative protein expression of $\beta 3$ integrin was determined by using densitometry and normalized to $\beta$-actin controls in each sample. (F) miR-30a-5p was lower in TNBC samples than in NTNBC samples. (G) miR-30a-5p was inversely correlated with $\beta 3$ integrin expression in breast cancer tissues $\left(r^{2}=0.47\right)$. ${ }^{*} \mathrm{P}<0.05,{ }^{* *} \mathrm{P}<0.01$.

mRNA expression of miR-30a-5p and $\beta 3$ integrin in TNBC patients (Fig. $1 G, r^{2}=0.47, P<0.05$; Pearson's correlation). These differences in mRNA and protein expression of $\beta 3$ integrin in breast cancer patients suggested probable post-transcriptional regulation by miR-30a-5p.

Knockdown of $\beta 3$ integrin results in the alteration of EMT markers. To investigate the role of $\beta 3$ integrin knockdown on the reversal of EMT phenotype of breast cancer cells, siRNAtargeting $\beta 3$ integrin was transfected into MDA-MB-231 cells. After 14 days of transfection, the morphology of $\beta 3$ integrinsilenced MDA-MB-231 was partially changed from elongated fibroblastoid to epithelial cobblestone-like appearance, with the cells appearing to grow in close contact with each other (Fig. 2A). The silencing of $\beta 3$ integrin in the MDA-MB-231 cells resulted in the elevation of epithelial marker E-cadherin and the downregulation of mesenchymal markers, including Zeb1 and vimentin, at mRNA and protein levels (Fig. 2B and $\mathrm{C}$ ).

We next investigated whether molecular alterations were present in E-cadherin and vimentin protein by immunostaining. Silencing of $\beta 3$ integrin showed that E-cadherin protein was significantly upregulated compared with the levels detected in control cells (Fig. 2D). To determine the role of $\beta 3$ integrin in actin cytoskeletal reorganization, cells with silenced $\beta 3$ integrin were stained for F-actin and vinculin and showed morphological changes, including formation of protrusions and destruction of actin filaments (Fig. 2E). These results suggest that the $\beta 3$ integrin is critical for the acquisition of EMT characteristics and that inhibition 
Table II. Clinicopathological characteristics and miR-30a-5p expression in breast cancer.

\begin{tabular}{|c|c|c|c|}
\hline Clinicopathological variables & Cases $(\%)$ & $\log _{2}$ (fold of repression) $($ mean $\pm S D)$ & P-value \\
\hline \multicolumn{4}{|l|}{ Age (years) } \\
\hline$\leq 45$ & $72(46.1)$ & $4.17 \pm 0.47$ & $0.268^{\mathrm{a}}$ \\
\hline$>45$ & $84(53.9)$ & $4.02 \pm 0.51$ & \\
\hline \multicolumn{3}{|l|}{ Molecular-based classification } & $0.006^{\mathrm{b}}$ \\
\hline TNBC & $45(28.8)$ & $2.47 \pm 0.39$ & \\
\hline NTNBC & $111(71.2)$ & $4.51 \pm 0.59$ & \\
\hline \multicolumn{3}{|l|}{ Tumor size (cm) } & $0.173^{\mathrm{b}}$ \\
\hline$\leq 2$ & $66(42.3)$ & $4.37 \pm 0.53$ & \\
\hline $2-5$ & $79(50.6)$ & $4.05 \pm 0.65$ & \\
\hline$>5$ & $11(7.1)$ & $3.71 \pm 0.24$ & \\
\hline \multicolumn{3}{|l|}{ Histological grade } & $0.003^{\mathrm{b}}$ \\
\hline I & $48(30.4)$ & $4.47 \pm 0.32$ & \\
\hline II & $83(52.5)$ & $4.16 \pm 0.53$ & \\
\hline III & $27(17.1)$ & $2.19 \pm 0.22$ & \\
\hline \multicolumn{3}{|l|}{ Clinical stage } & $0.047^{\mathrm{b}}$ \\
\hline I, II & $113(72.4)$ & $4.52 \pm 0.42$ & \\
\hline III & $36(23.1)$ & $4.18 \pm 0.36$ & \\
\hline IV & $7(4.5)$ & $3.89 \pm 0.32$ & \\
\hline \multicolumn{3}{|l|}{ Positive lymph nodes } & $0.001^{\mathrm{b}}$ \\
\hline 0 & $64(41.0)$ & $4.83 \pm 0.32$ & \\
\hline $1-3$ & $51(32.7)$ & $4.31 \pm 0.64$ & \\
\hline$\geq 4$ & $41(26.3)$ & $4.19 \pm 0.22$ & \\
\hline \multicolumn{3}{|l|}{ Survival } & $0.003^{\mathrm{b}}$ \\
\hline Alive & $114(73.1)$ & $5.49 \pm 0.52$ & \\
\hline Deceased & 42 (26.9) & $2.86 \pm 0.36$ & \\
\hline
\end{tabular}

andependent-samples t-test. ${ }^{\mathrm{b}}$ One-way ANOVA test.

of $\beta 3$ integrin was able to reverse the EMT phenotype of breast cancer cells.

miR-30a-5p directly targets the $\beta 3$ integrin 3' untranslated region ( $\left.3^{\prime}-U T R\right)$. To investigate the direct effects of miR-30a-5p on $\beta 3$ integrin expression in breast cancer cell lines, we performed miR-30a-5p overexpression experiments in MDA-MB-231 cells. Infection of miR-30a-5p mimic into MDA-MB-231 cells increased miR-30a-5p levels (data not shown). Ectopic overexpression of miR-30a-5p resulted in a significantly decrease in $\beta 3$ integrin $\mathrm{mRNA}$ levels, as determined by qRT-PCR (Fig. 3A). This suppression was also found at the protein level, as observed by western blot analysis (Fig. 3B). On the contrary, miR-30a-5p inhibitor restored $\beta 3$ integrin expression in a dose-dependent manner (Fig. 3C). Online programs (TargetScan, miRBase and PicTar) revealed that a region in the 65-72 $\beta 3$ integrin 3'-UTR had a perfect complementary matching region in the seed sequence of miR-30a-5p (Fig. 3D and E). To confirm that the silencing of $\beta 3$ integrin expression is consequent to miR-30a-5p targeting of the 3'-UTR in $\beta 3$ integrin transcript, the complete 3'-UTR of $\beta 3$ integrin and corresponding mutant counterparts were cloned into pGL3 firefly luciferase-containing vector (Fig. 3F).
HEK-293T cells were cotransfected with the firefly luciferasecontaining vector, Renilla luciferase-containing vector, and pre-miR-30a-5p. The results revealed a strong repression of luciferase activity after transfection with wild-type 3'-UTR of $\beta 3$ integrin, but not in cells with mutant 3'-UTR (Fig. 3G).

miR-30a-5p reduces the adhesion capacity of TNBC cells. Ectopic miR-30a-5p also inhibited the proliferation rate of MDA-MB-231 and MDA-MB-486 cells by inducing cell cycle arrest at the G0/G1 phase, as shown by the MTT assay and flow cytometry (data not shown). To eliminate the potential cofounding effect of cell proliferation on cell migration and invasion, the Transwell experiment and scratch assay were conducted in the presence of mitomycin $\mathrm{C}$ to subsequently arrest cell proliferation.

$\beta 3$ integrin signaling is associated with many cellular functions. Integrin-mediated interactions with the extracellular matrix (ECM) are required for attachment, cytoskeletal organization, mechanosensing, migration, proliferation, differentiation and survival of cells. To test whether miR-30a-5p functionally behaves as a tumor suppressor by targeting $\beta 3$ integrin, we stably overexpressed miR-30a-5p in MDA-MB-231 and MDA-MB-486 using a lentiviral vector. 
A
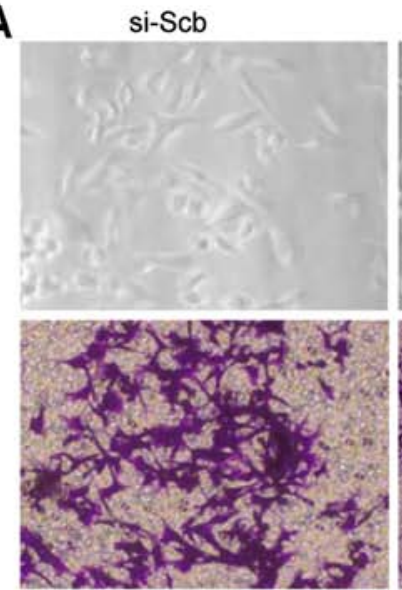

B

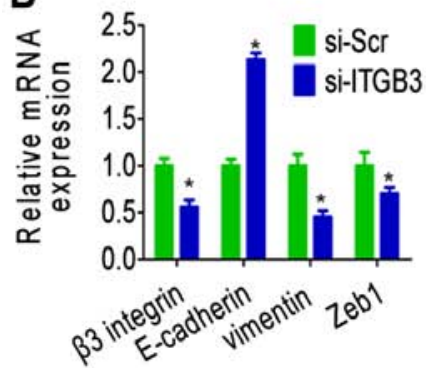

si-ITGB3
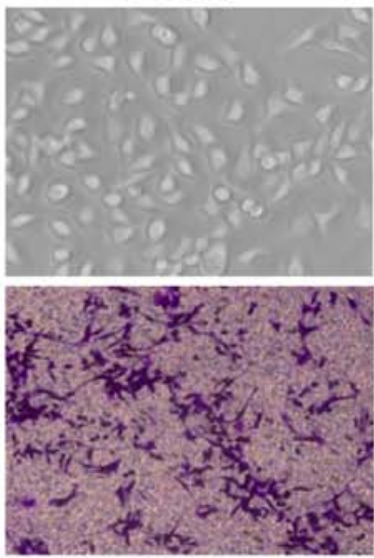

C

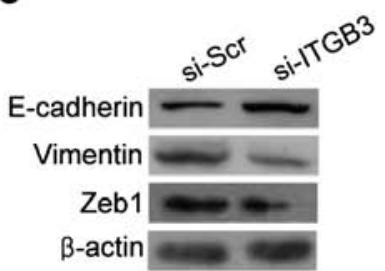

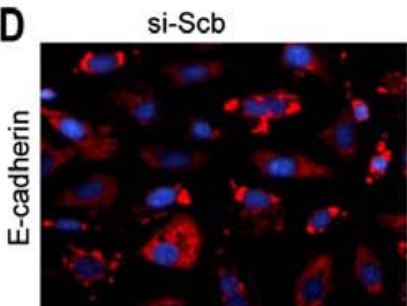
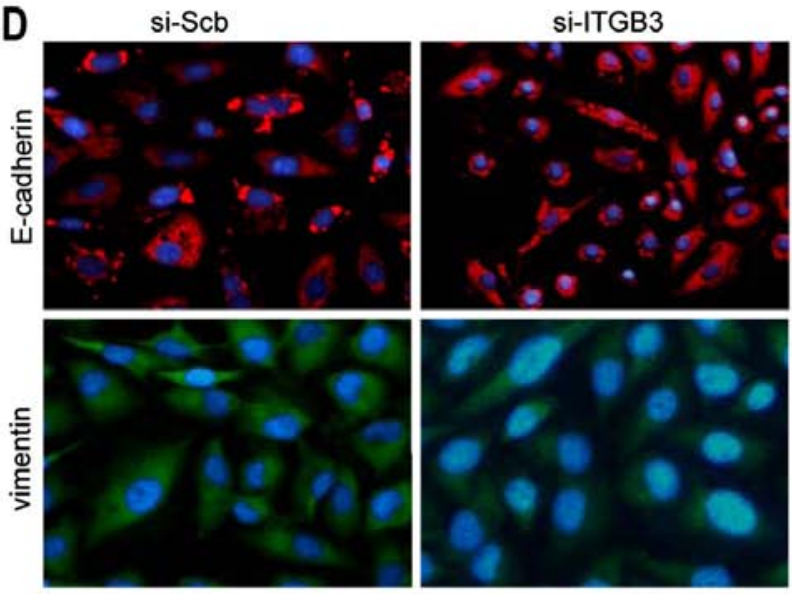

E

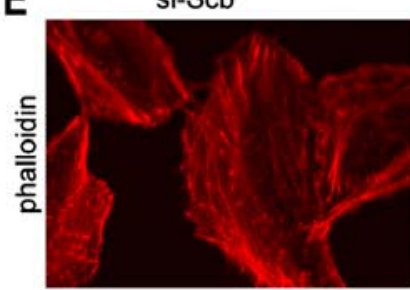

si-ITGB3

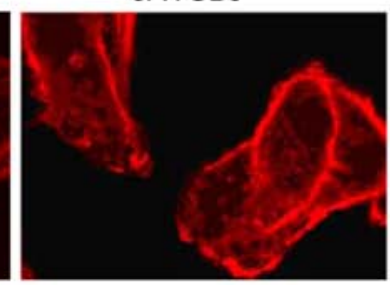

Figure 2. Inhibitory effect on EMT in breast cancer cells by knockdown of $\beta 3$ integrin. (A) The morphology of MDA-MB-231 cells changed from fibroblastoid to epithelial-like appearance after silencing $\beta 3$ integrin (upper panel). Downregulation of $\beta 3$ integrin facilitated the adhesion of MDA-MB-231 cells in vitro (lower panel). (B) Silencing of $\beta 3$ integrin in MDA-MB-231 resulted in the upregulation of E-cadherin expression and downregulation of Zeb1 expression. Vimentin was assessed by real-time RT-PCR. (C) Western blot analysis was performed to determine E-cadherin, vimentin and Zeb-1 expressions. (D) Fluorescence microscopic staining of E-cadherin and vimentin was performed in MDA-MB-231 cells. Nuclear DNA was stained with DAPI. (E) miR-30a-5p-overexpressing cells exhibited protrusion formation and actin filament destruction compared with uninfected cells. "P<0.05.
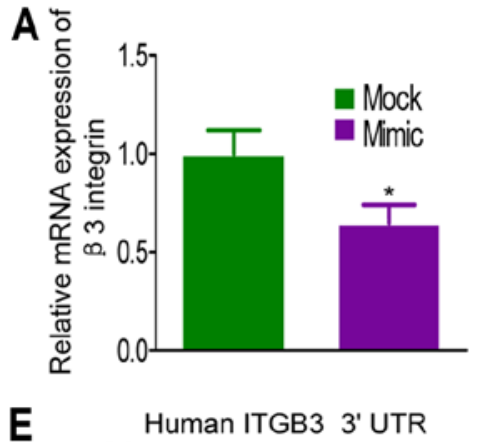

B

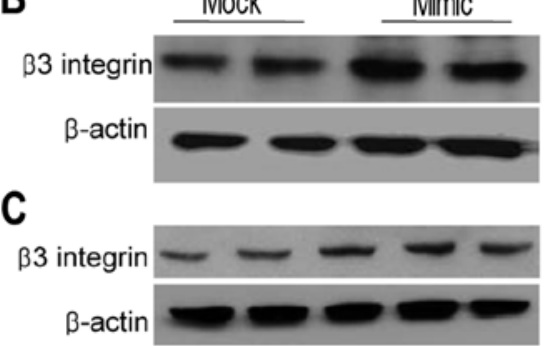

$\mathbf{E}$
D

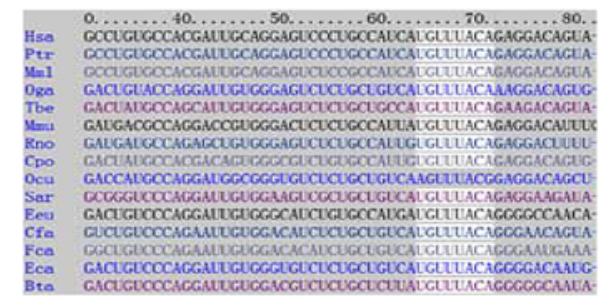
Human ITGB3 3' UTR $0.5 \mathrm{k}$ $1 \mathrm{k}$ $1.5 \mathrm{k}$ miR-30a-5p let-7 miR-125a let-7 miR-150 miR-221 miR-19b miR-93

$\mathbf{F}$ hsa-miR-30a-5p 3'-CCUGCCAUCAUGUUUACAGUCG-5' 3' UTR-ITGB3-WT 5'-GUCAGCUCCUACAAAUGUGAGG -3' 3'-UTR-ITGB3-MUT 5'-GUCAGCUCCUACAGAUGAGAGG -3'

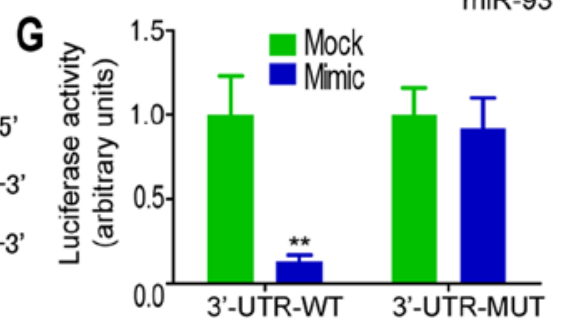

Figure 3. $\beta 3$ integrin is specifically targeted by miR-30a-5p. (A) The potential for miR-30a-5p-mediated silencing of $\beta 3$ integrin by the predicted miRNAs was tested in MDA-MB-231 cells by qRT-PCR. (B) miR-30a-5p overexpression reduced the $\beta 3$ integrin protein expression of MDA-MB-231 cells. (C) Different transfection concentration of miR-30a-5p gradually increased $\beta 3$ integrin expression in MDA-MB-231 cells. (D and E) Putative binding sites for miR-30a-5p were predicted by using the PicTar and TargetScan algorithms. (F) Schematic representation of the predicted target site of miR-30a-5p in the wild-type (WT) 3'-UTR of $\beta 3$ integrin and mutant (MUT) $\beta 3$ integrin 3'-UTR. Seed matching sequence is indicated. (G) Ectopic miR-30a-5p expression inhibited WT, but not the activity of MUT $\beta 3$ integrin $3 '-U T R,{ }^{*} \mathrm{P}<0.05,{ }^{* *} \mathrm{P}<0.01$. 

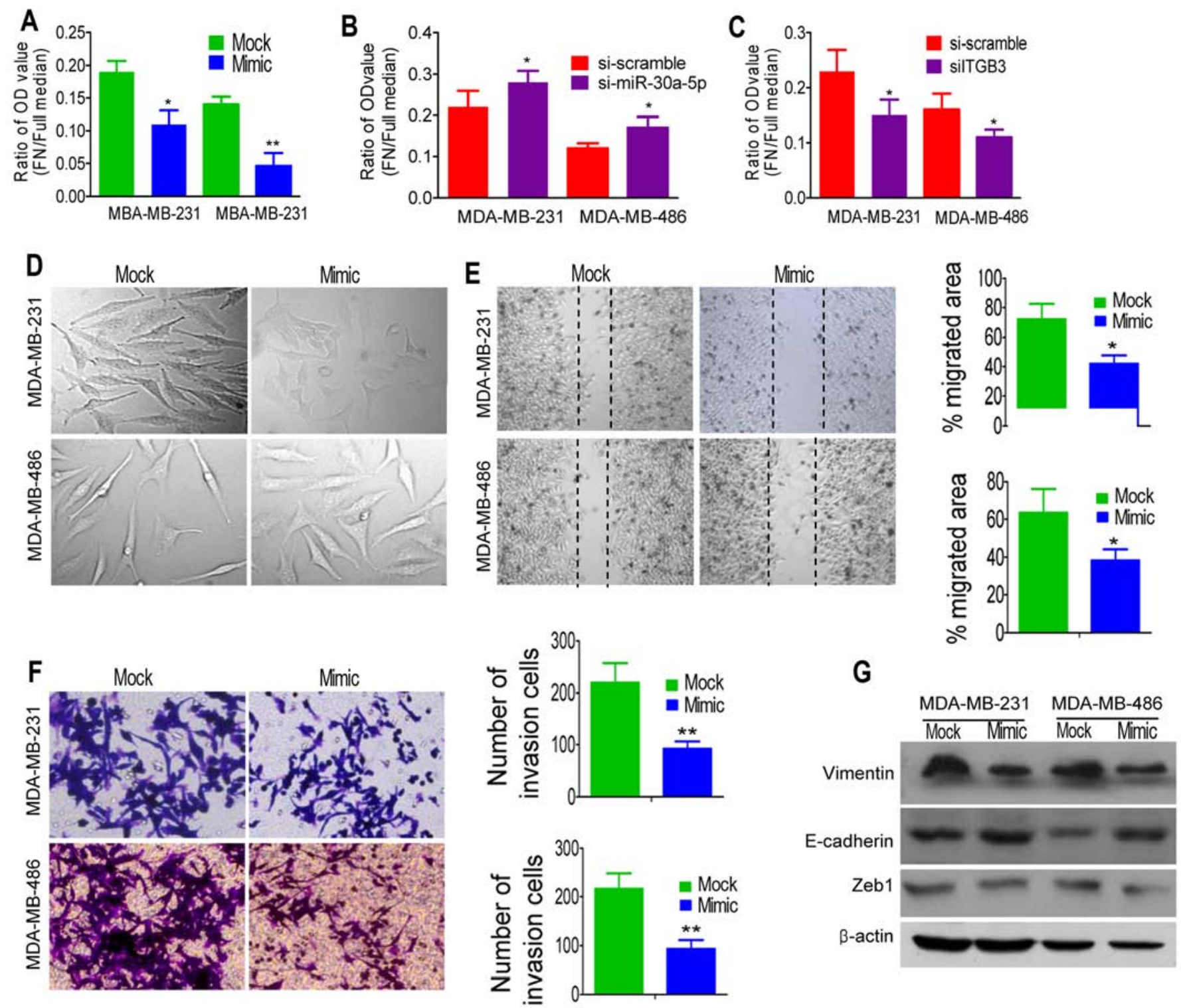

Figure 4. miR-30a-5p affects the properties of adhesion, migration and invasion in TNBC cells. (A) Ectopic miR-30a-5p suppressed cellular adhesion of TNBC cells. (B) Downregulation of miR-30a-5p facilitated TNBC cell adhesion in vitro. (C) Silencing of $\beta 3$ integrin reduced cell adhesion. (D) Morphological changes in miR-30a-5p-overexpressing cells compared with control cells. (E) Effects of ectopic expression of miR-30a-5p on the migration of TNBC cells. (F) Effects of ectopic expression of miR-30a-5p on the invasion of TNBC cells. (G) EMT-related markers show different expressions in TNBC cells with or without miR-30a-5p overexpression. ${ }^{*} \mathrm{P}<0.05,{ }^{* *} \mathrm{P}<0.01$.

We focused on the effect of miR-30a-5p on the adhesion of TNBC cells by planting cells in plates coated with fibronectin, which could bind to a $\beta 3$ integrin receptor expressed on the surface of tumor cells (18). As shown in Fig. 4A, the adhesion ability of MDA-MB-231 and MDA-MB-486 cells with miR-30a-5p overexpression showed significantly reduced adherence compared with the control cells. On the contrary, the adhesion ability of cells was lower when miR-30a-5p was inhibited using siRNA than when scramble siRNA was used (Fig. 4B). Moreover, reduction occurrence after transfection with siRNA directed against $\beta 3$ integrin (siITGB3) elucidated the significance of reduced $\beta 3$ integrin in overexpressed miR-30a-5p cells (Fig. 4C).

Ectopic expression of miR-30a-5p suppresses cell migration and invasion in vitro. To test whether miR-30a-5p overexpression suppressed tumor migration and invasion, we first examined the morphological changes in miR-30a-5poverexpressed cells. As shown in Fig. 4D, MDA-MB-231 cells overexpressing miR-30a-5p exhibited epithelial morphology. Cells overexpressing miR-30a-5p showed a non-aggressive appearance and depressed adhesion of cell to fibronectin. Thus, we hypothesized that miR-30a-5p could suppress the migration and invasive behavior of TNBC cancer cells. Then, wound scratch and Transwell assay were employed to detect the migration and invasion after miR-30a-5p manipulation, respectively. We found that miR-30a-5p could significantly suppress migration (Fig. 4E) and invasion (Fig. 4F) in TNBC cells lines. To further investigate whether the inhibitory effect of miR-30a-5p on migration and invasion was mediated by mesenchymal to epithelia transition (MET), we examined the expression of several MET markers. As expected, miR-30a-5p 
A

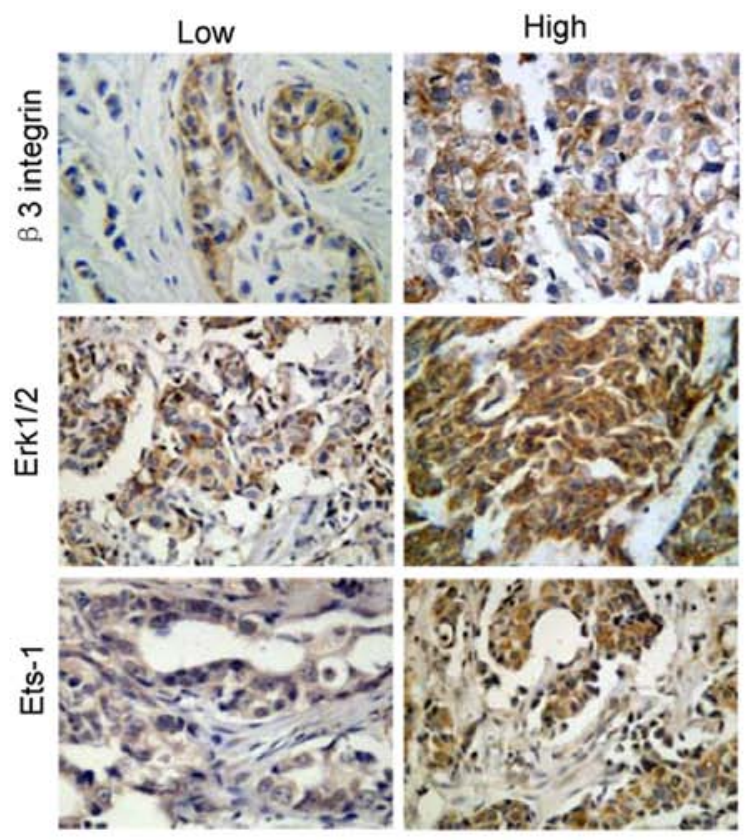

B

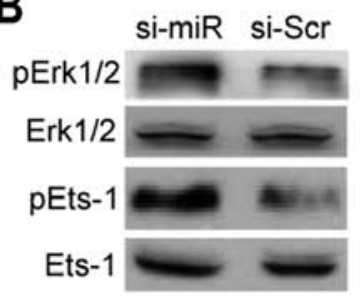

D

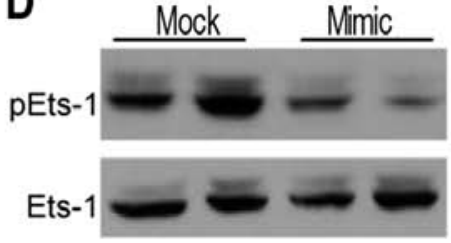

E

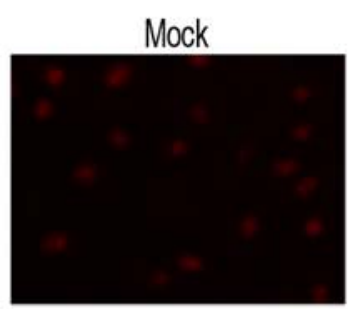

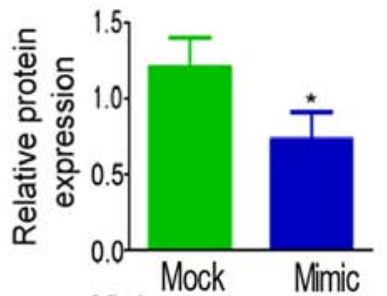
Mimic

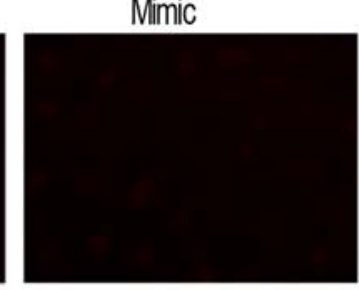

F

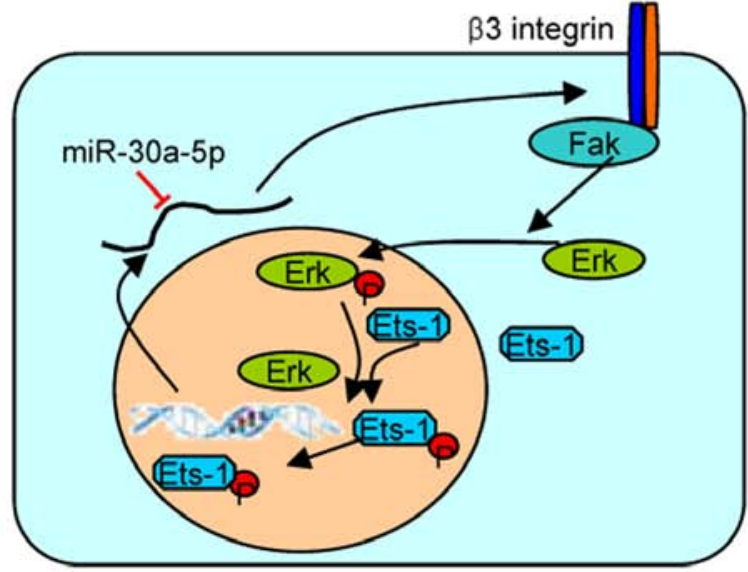

Figure 5. Images of $\beta 6$ and Ets-1 immunohistochemistry staining in human breast cancer samples. (A) Immunohistochemistry photomicrographs of $\beta 6$ integrin, Erk1/2, Ets-1 and $\beta 6$ integrin staining were observed predominantly in the cell membrane. Erk1/2 and Ets-1 mainly exhibited nuclear and cytoplasmic immunostaining in tumor cells. (B) Cellular proteins were isolated for western blot detection of indicated signal proteins. Inhibition of Erk1/2 blocks Ets-1 phosphorylation in MDA-M-231 cells with miR-30a-5p overexpression. (C) Inhibition of Erk1/2 strongly suppressed Erk1/2 and pEts-1 phosphorylation compared with the control (DMSO). (D) Ectopic miR-30a-5p enhanced the expression of pEts-1. (E) miR-30a-5p overexpression mediated the nuclear translocation of phosphorylated Ets-1. (F) The working model was as follows: a $\beta 3$ integrin-driven feed-forward Erk/Ets-1-activation signaling loop, which was required for the maintenance of self-renewal and tumorigenicity of TNCBs, was suspended upon overexpression of miR-30a-5p. ${ }^{*} \mathrm{P}<0.05$.

Table III. Expression correlation between $\beta 6$ integrin and Erk1/2 or transcriptional factor Ets-1.

\begin{tabular}{|c|c|c|c|c|c|c|}
\hline \multirow{2}{*}{$\begin{array}{l}\beta 6 \text { integrin } \\
\text { expression }\end{array}$} & \multicolumn{3}{|c|}{ Erk1/2 expression } & \multicolumn{3}{|c|}{ Ets-1 expression } \\
\hline & Negative or low & High & P-value & Negative or low & High & P-value \\
\hline Negative or low & 55 & 41 & $<0.05$ & 63 & 38 & $<0.01$ \\
\hline High & 22 & 38 & & 22 & 33 & \\
\hline
\end{tabular}

overexpression increased the expression level of E-cadherin and decreased the expression levels of vimentin and Zeb1 (Fig. 4G). These observations suggested that ectopic expression of miR-30a-5p was able to impede migration and invasion mediated by MET in vitro.

miR-30a-5p interrupts the $\beta 3$ integrin-Erk-Ets-1 network in triple-negative breast cancer. Integrins elicit a series of transduction events that regulate cell cycle progression and apoptosis in a process known as 'outside-in' signaling. The 'outside-in' $\beta 3$ integrin-mediated signaling proceeds primarily via the Erk1/2 MAPK pathway in MDA-MB-231 breast cancer cells. Immunohistochemical staining of $\beta 3$ integrin, Erk1/2, and Ets-1 in breast cancer and normal tissues are shown in Fig. 5A. Results indicated that expression of $\beta 3$ and Erk1/2 $(\mathrm{P}<0.05)$ and $\beta 6$ and Ets- $1(\mathrm{P}<0.01)$ were positively correlated, as shown in Table III.

To further confirm the association between Erk1/2-EtS phosphorylation and miR-30a-5p inhibition, we collected extracts from si-miR-30a-5p MDA-MB-231 cells. Western blot analysis of the extracts showed a specific enhancement of pErk1/2 (Fig. 5B). As the activities of Ets-1 were enhanced by 
Erk1/2-mediated phosphorylation at threonine 38 for Ets-1 (26), we further examined the phosphorylation levels of Ets-1, showing Erk1/2 activation correlated well with the elevated phosphorylation levels of pEts-1, whereas PD98059 (Erk pathway inhibitor) markedly lowered pEts-1 levels (Fig. 5C). However, Ets-1 phosphorylation was also enhanced (Fig. 5D) with obvious nuclear translocation in ectopic miR-30a-5pexpressing MDA-MB-231 cells (Fig. 5E). Similar correlations between miR-30a-5p overexpression and reduced Erk1/2-Ets phosphorylation were found in MDA-MB-486 cells (data not shown). Thus, $\beta 3$ integrin induced ERK1/2 phosphorylation, thereby triggering the activation of Ets- 1 transcription factors leading to $\beta 3$ integrin upregulation, whereas miR-30a-5p interdicted the positive feedback loop (Fig. 5F).

\section{Discussion}

TNBC has a high incidence of early relapse and metastasis and contributes to poor clinical outcomes. Treatment options are limited for TNBC because endocrinotherapy and targeted therapy, which aim directly at human epidermal growth factor receptor-2, are ineffective. Interactions between cells and ECM convey micro-environmental cues that influence cell proliferation, differentiation, adhesion and migration $(19,20)$. Thus, targeting cell-ECM interactions can become a potential component of an oncologist's therapeutic arsenal as a novel therapy for TNBC. Integrins, a family of cell adhesion molecules, are involved in a wide range of cell-ECM and cell-cell interactions. Basement membrane proteins interact with mammary epithelial cells via integrins and transmembrane proteoglycans and syndecan, which all couple to the cytoskeleton and assemble signaling platforms to control cell function (21). Integrin and associated intracellular signaling effector expression levels and/or activity are modified in TNBCs, thereby suggesting that the adhesion machinery has a role in malignant transformation and tumor progression $(7,22)$.

Integrin adhesion receptors modulate cell functions, including cell proliferation; hypodermic injection of breast cancer cells stably transfected with $\beta 1$ integrin into athymic nude mice resulted in decreased size and weight of subcutaneous xenograft tumors (23). Moreover, $\beta 1$ integrins support outgrowth of metastatic colonies in the lung. In the present study, we demonstrated that ectopic expression of $\beta 3$ integrin attenuated the proliferation and induced cell cycle arrest at G0/G1 phase in breast cancer cells. This confirms and extends earlier reports where outgrowth of breast cancer cells arrest also showed the role of $\beta 3$ integrin signaling in the modulation of cell proliferation of breast cancer (24).

$\beta 3$ integrin has been detected in tumor tissues from patients with melanoma, breast cancer and its expression is particularly pronounced in metastatic tissue $(7,25,26)$. TNBC is a highly aggressive subgroup of breast cancer and currently lacks definite molecular targets for effective targeted therapies. Among the 156 tissue specimens, 57 (36.5\%) were positive for $\beta 3$ expression. A significant statistical difference existed between TNBC and NTNBC groups. Previous studies exploited $\beta 3$ integrin as a therapeutic target for treating TNBC by delivering siRNA $(7,8)$. Breast cancer cell adhesion and spread are triggered by the contact between $\beta 3$ integrin and fibrinogen. Blocking $\beta 3$ integrin with antibodies and siRNA leads to significantly lower adhesion of MDA-MB-231 cells to fibronectin (27). EMT is a crucial procedure in tumor metastasis; therefore, prevention of EMT represents a very promising therapeutic strategy to prevent tumor metastasis $(28,29)$. In the present study, reduced expression of $\beta 3$ integrin could cause morphological changes in MDA-MB-231 cells, i.e., from fibroblastoid cells to epithelial-like cells with weak invasive capacity. Silencing of $\beta 3$ integrin promoted MDA-MB-231 cell migration due to the change in the amount of organized actin cytoskeleton. This finding is significative because targeting TNBC by $\beta 3$ integrin might provide a valuable tool in developing new therapeutic avenues against metastasis.

MiRNAs negatively regulate EMT-related genes at the post-transcriptional level and play critical roles in cancer metastasis. In the present study, we reported that miR-30a-5p is associated with the regulation of EMT. Aberrant expression of miRNA has been implicated in the deregulation of integrin expression and activity. MiRNA-130b suppresses migration and invasion of colorectal cancer cells through downregulation of $\beta 1$ integrin (30). We found that overexpression of miR-30a-5p in breast cancer cells significantly suppressed $\beta 3$ integrin in vitro, whereas, miR-30a-5p was inversely correlated with $\beta 3$ integrin expression in breast cancer tissues. Computational prediction by using the TargetScan software revealed an evolutionarily conserved region in the $\beta 3$ integrin 3'-UTR mRNA, which has a perfect complementary matching region to the seed sequence of the miR-30a-5p. Luciferase activity assay indicated that miR-30a-5p could bind to the 3'-UTR sequence of $\beta 3$ integrin mRNA, whereas $\beta 3$ integrin is a direct target of miR-30a-5p.

Many cellular responses to soluble growth factors, such as EGF, PDGF, LPA and thrombin, depend on the adhesion of cells to substrates via integrins (31). The integrin family represents major receptors that mediate adhension to the ECM and trigger critical intracellular signaling pathways involved in the invasion and migration (32). Previous studies show that the presence of a positive feedback loop between $\beta 3$ integrin and Ets-1 $(33,34)$. Thus, $\beta 3$ integrin might be a novel therapeutic target. Results from our study indicated that the expression level of $\beta 3$ and Ets-1 was associated with differentiation, TNM stage, and breast cancer classification. Correlation analysis showed that the expression of $\beta 3$ and Ets-1 were positively correlated. To verify the potential impact of ectopic miR-30a-5p on phosphorylation Ets-1, the activation of the ERK/Est-1 was determined. Overexpression of miR-30a-5p displayed reduced phosphorylation of Erk1/2 and Est-1, and weakened the nuclear localization of Ets-1 in MDA-MB-231 cells. Furthermore, inhibition of Erk attenuated the silence-mediated upregulation of pEts-1 in miR-30a-5p.

In conclusion, $\beta 3$ integrin contributed to cancer progression in TNBCs by regulating Erk1/2 and Est-1, which drive malignant cell behavior. This regulation can be exploited in therapeutic strategies to inhibit cancer progression. The present study showed that miR-30a-5p, as a tumor suppressor, is a regulator of $\beta 3$ integrin replication in breast cancer. This validates the concept of targeting the cross link of $\beta 3$ integrin/ Erk/Ets-1 network as a novel and promising modality to treat TNBCs. 


\section{Acknowledgements}

The present study was funded by grants from the National Nature Scientific Foundation of China (30901779 and 81471048) and the Natural Science Foundation of Shandong Province (ZR2015HL064).

\section{References}

1. Taylor MA, Davuluri G, Parvani JG, Schiemann BJ, Wendt MK Plow EF, Schiemann WP and Sossey-Alaoui K: Upregulated WAVE3 expression is essential for TGF- $\beta$-mediated EMT and metastasis of triple-negative breast cancer cells. Breast Cancer Res Treat 142: 341-353, 2013.

2. Barcus CE, Keely PJ, Eliceiri KW and Schuler LA: Stiff collagen matrices increase tumorigenic prolactin signaling in breast cancer cells. J Biol Chem 288: 12722-12732, 2013.

3. Wang Y, Shenouda S, Baranwal S, Rathinam R, Jain P, Bao L, Hazari S, Dash S and Alahari SK: Integrin subunits alpha5 and alpha6 regulate cell cycle by modulating the chk1 and Rb/E2F pathways to affect breast cancer metastasis. Mol Cancer 10: 84, 2011.

4. Felding-Habermann B, O'Toole TE, Smith JW, Fransvea E, Ruggeri ZM, Ginsberg MH, Hughes PE, Pampori N, Shattil SJ, Saven A, et al: Integrin activation controls metastasis in human breast cancer. Proc Natl Acad Sci USA 98: 1853-1858, 2001.

5. Salvo E, Garasa S, Dotor J, Morales X, Peláez R, Altevogt P and Rouzaut A: Combined targeting of TGF- $\beta 1$ and integrin $\beta 3$ impairs lymph node metastasis in a mouse model of non-smallcell lung cancer. Mol Cancer 13: 112, 2014.

6. Li N, Zhang JP, Guo S, Min J, Liu LL, Su HC, Feng YM and Zhang HL: Down-regulation of $\beta 3$-integrin inhibits bone metastasis of small cell lung cancer. Mol Biol Rep 39: 3029-3035, 2012.

7. Parvani JG, Gujrati MD, Mack MA, Schiemann WP and Lu ZR: Silencing $\beta 3$ integrin by targeted ECO/siRNA nanoparticles inhibits EMT and metastasis of triple-negative breast cancer. Cancer Res 75: 2316-2325, 2015.

8. Huveneers S and Danen EH: Adhesion signaling - crosstalk between integrins, Src and Rho. J Cell Sci 122: 1059-1069, 2009

9. Raymond K, Faraldo MM, Deugnier MA and Glukhova MA: Integrins in mammary development. Semin Cell Dev Biol 23: 599-605, 2012

10. Harquail J, Benzina S and Robichaud GA: MicroRNAs and breast cancer malignancy: an overview of miRNA-regulated cancer processes leading to metastasis. Cancer biomarkers: section. Dis Markers 11: 269-280, 2012.

11. de Krijger I, Mekenkamp LJ, Punt CJ and Nagtegaal ID: MicroRNAs in colorectal cancer metastasis. J Pathol 224: 438-447, 2011.

12. Shi M and Guo N: MicroRNA expression and its implications for the diagnosis and therapeutic strategies of breast cancer. Cancer Treat Rev 35: 328-334, 2009.

13. Zhang N, Wang X, Huo Q, Sun M, Cai C, Liu Z, Hu G and Yang Q: MicroRNA-30a suppresses breast tumor growth and metastasis by targeting metadherin. Oncogene 33: 3119-3128, 2014.

14. Kao CJ, Martiniez A, Shi XB, Yang J, Evans CP, Dobi A, deVere White RW and Kung HJ: miR-30 as a tumor suppressor connects EGF/Src signal to ERG and EMT. Oncogene 33: 2495-2503, 2014.

15. Baraniskin A, Birkenkamp-Demtroder K, Maghnouj A, Zöllner H, Munding J, Klein-Scory S, Reinacher-Schick A, Schwarte-Waldhoff I, Schmiegel W and Hahn SA: MiR-30a-5p suppresses tumor growth in colon carcinoma by targeting DTL. Carcinogenesis 33: 732-739, 2012.
16. Hu G, Chong RA, Yang Q, Wei Y, Blanco MA, Li F, Reiss M, $\mathrm{Au}$ JL, Haffty BG and Kang Y: MTDH activation by $8 \mathrm{q} 22$ genomic gain promotes chemoresistance and metastasis of poorprognosis breast cancer. Cancer Cell 15: 9-20, 2009.

17. Heckman KL and Pease LR: Gene splicing and mutagenesis by PCR-driven overlap extension. Nat Protoc 2: 924-932, 2007.

18. Knowles LM, Gurski LA, Engel C, Gnarra JR, Maranchie JK and Pilch J: Integrin $\alpha v \beta 3$ and fibronectin upregulate Slug in cancer cells to promote clot invasion and metastasis. Cancer Res 73: 6175-6184, 2013.

19. Hynes RO: Integrins: Bidirectional, allosteric signaling machines. Cell 110: 673-687, 2002.

20. Hynes RO: Targeted mutations in cell adhesion genes: What have we learned from them? Dev Biol 180: 402-412, 1996.

21. Morgan MR, Humphries MJ and Bass MD: Synergistic control of cell adhesion by integrins and syndecans. Nat Rev Mol Cell Biol 8: 957-969, 2007.

22. Gehler S, Ponik SM, Riching KM and Keely PJ: Bi-directional signaling: Extracellular matrix and integrin regulation of breast tumor progression. Crit Rev Eukaryot Gene Expr 23: 139-157, 2013.

23. Truong HH, Xiong J, Ghotra VP, Nirmala E, Haazen L, Le Dévédec SE, Balcioğlu HE, He S, Snaar-Jagalska BE, Vreugdenhil E, et al: $\beta 1$ integrin inhibition elicits a prometastatic switch through the TGF $\beta$-miR-200-ZEB network in E-cadherinpositive triple-negative breast cancer. Sci Signal 7: ra15, 2014.

24. Sumimoto S, Muramatsu R, Fujii S and Yamashita T: Vascular endothelial cells promote cortical neurite outgrowth via an integrin $\beta 3$-dependent mechanism. Biochem Biophys Res Commun 450: 593-597, 2014.

25. Nasulewicz-Goldeman A, Uszczyńska B, Szczaurska-Nowak K and Wietrzyk J: siRNA-mediated silencing of integrin $\beta 3$ expression inhibits the metastatic potential of B16 melanoma cells. Oncol Rep 28: 1567-1573, 2012.

26. Li A, Guo Q, Kim C, Hu W and Ye F: Integrin alphaII b tail distal of GFFKR participates in inside-out alphaII $b$ beta3 activation. J Thromb Haemost 12: 1145-1155, 2014.

27. Beauvais DM and Rapraeger AC: Syndecan-1-mediated cell spreading requires signaling by alphavbeta 3 integrins in human breast carcinoma cells. Exp Cell Res 286: 219-232, 2003.

28. Kudo-Saito C, Shirako H, Takeuchi T and Kawakami Y: Cancer metastasis is accelerated through immunosuppression during Snail-induced EMT of cancer cells. Cancer Cell 15: 195-206, 2009.

29. Spaderna S, Schmalhofer O, Hlubek F, Berx G, Eger A, Merkel S, Jung A, Kirchner T and Brabletz T: A transient, EMT-linked loss of basement membranes indicates metastasis and poor survival in colorectal cancer. Gastroenterology 131: 830-840, 2006.

30. Augoff K, Das M, Bialkowska K, McCue B, Plow EF and SosseyAlaoui $\mathrm{K}$ : miR-31 is a broad regulator of $\beta 1$-integrin expression and function in cancer cells. Mol Cancer Res 9: 1500-1508, 2011.

31. Frijns E, Sachs N, Kreft M, Wilhelmsen K and Sonnenberg A: EGF-induced MAPK signaling inhibits hemidesmosome formation through phosphorylation of the integrin \{beta\}4. J Biol Chem 285: 37650-37662, 2010.

32. Wei Y, Tang CH, Kim Y, Robillard L, Zhang F, Kugler MC and Chapman HA: Urokinase receptors are required for alpha 5 beta 1 integrin-mediated signaling in tumor cells. J Biol Chem 282: 3929-3939, 2007.

33. Sato T and Miwa A: Ets-1 and integrin beta 3 for lung metastasis from colorectal cancer. APMIS 110: 347-353, 2002.

34. Rothhammer T, Hahne JC, Florin A, Poser I, Soncin F, Wernert N and Bosserhoff AK: The Ets-1 transcription factor is involved in the development and invasion of malignant melanoma. Cell Mol Life Sci 61: 118-128, 2004. 